\title{
Effect of cellular and extracellular pathology assessed by T1 mapping on regional contractile function in hypertrophic cardiomyopathy
}

Peter P. Swoboda ${ }^{1 *}+\mathbb{D}$, Adam K. McDiarmid ${ }^{1 \dagger}$, Bara Erhayiem, Graham R. Law², Pankaj Garg ${ }^{1}$, David A. Broadbent ${ }^{1,3}$,


John P. Greenwood ${ }^{1}$ and Sven Plein ${ }^{1}$

\begin{abstract}
Background: Regional contractile dysfunction is a frequent finding in hypertrophic cardiomyopathy $(\mathrm{HCM})$. We aimed to investigate the contribution of different tissue characteristics in HCM to regional contractile dysfunction.

Methods: We prospectively recruited 50 patients with HCM who underwent cardiovascular magnetic resonance (CMR) studies at 3.0 T including cine imaging, T1 mapping and late gadolinium enhancement (LGE) imaging. For each segment of the American Heart Association model segment thickness, native T1, extracellular volume (ECV), presence of LGE and regional strain (by feature tracking and tissue tagging) were assessed. The relationship of segmental function, hypertrophy and tissue characteristics were determined using a mixed effects model, with random intercept for each patient.
\end{abstract}

Results: Individually segment thickness, native T1, ECV and the presence of LGE all had significant associations with regional strain. The first multivariable model (segment thickness, LGE and ECV) demonstrated that all strain parameters were associated with segment thickness $(P<0.001$ for all) but not ECV. LGE (Beta 2.603, $P=0.024)$ had a significant association with circumferential strain measured by tissue tagging.

In a second multivariable model (segment thickness, LGE and native T1) all strain parameters were associated with both segment thickness $(P<0.001$ for all) and native T1 $(P<0.001$ for all) but not LGE.

Conclusion: Impairment of contractile function in HCM is predominantly associated with the degree of hypertrophy and native T1 but not markers of extracellular fibrosis (ECV or LGE). These findings suggest that impairment of contractility in HCM is mediated by mechanisms other than extracellular expansion that include cellular changes in structure and function. The cellular mechanisms leading to increased native T1 and its prognostic significance remain to be established.

Keywords: Hypertrophic cardiomyopathy, T1 mapping, Extracellular volume, Strain, Tissue tagging, Feature tracking

\footnotetext{
* Correspondence: p.swoboda@leeds.ac.uk

${ }^{\dagger}$ Equal contributors

'Multidisciplinary Cardiovascular Research Centre (MCRC) \& Leeds Institute of Cardiovascular and Metabolic Medicine, University of Leeds, Clarendon Way, Leeds LS2 9JT, UK

Full list of author information is available at the end of the article
} 


\section{Background}

Hypertrophic cardiomyopathy (HCM) is commonly defined as a disease of hypertrophy of the left ventricle $(\mathrm{LV})$ in the absence of another cardiac or systemic cause [1]. Various patterns of hypertrophy are recognised [2] including widespread, asymmetric or eccentric myocardial thickening although most patients have a significant proportion of myocardium that is spared from overt hypertrophy [3].

HCM is most commonly caused by mutation in genes encoding proteins within the unit of myocardial contraction, the sarcomere [4]. The mechanisms that lead from dysfunction of the sarcomere to overt hypertrophy are complex and not as yet fully understood. Potential mechanisms include impaired calcium cycling, interstitial fibrosis, disturbed biomechanical stress sensing and microvascular dysfunction [5].

Previous studies predominantly performed with echocardiography speckle tracking have shown that there is widespread variation in myocardial contractility throughout the ventricle in HCM. Regional impairment of contractility is predominantly related to the extent of hypertrophy and presence of replacement fibrosis [6, 7]. Current guidelines therefore recommend that strain imaging could be used to investigate unexplained left ventricular hypertrophy that is not diagnostic of HCM $[1,8]$. Alternative methods to echocardiography for the assessment of regional strain are cardiovascular magnetic resonance $(\mathrm{CMR})$ tagging $[9,10]$ and post processing feature tracking (FT) [11-13] which are both highly reproducible and show good agreement.

Using CMR it is also possible to assess the tissue characteristics of the HCM phenotype. After administration of gadolinium based contrast agent, which is exclusively extracellular, late gadolinium enhancement (LGE) imaging allows identification of areas of focal fibrosis. Histological validation has suggested that the presence of LGE in HCM reflects replacement fibrosis [14], which is progressive over the course of the disease [15] and is associated with an adverse prognosis $[16,17]$.

LGE is a qualitative technique relying upon contrast between tissue with and without fibrosis, and is therefore of limited use in the detection of diffuse fibrosis. For this purpose T1 mapping techniques are used that give a quantitative pixel-wise map of myocardial $\mathrm{T} 1$ values. This can be performed without contrast (native $\mathrm{T} 1$ ), or by measuring both pre and post contrast T1maps to compute pixel-wise map of extracellular volume fraction (ECV \%) [18]. ECV has been validated in surgical samples in HCM and has been shown to correspond to the histologically measured extent of fibrosis [19, 20]. Native $\mathrm{T} 1$ is influenced by several factors including extracellular space expansion (as is ECV) but also intracellular iron, lipid and water content $[21,22]$. Native T1 has also been shown to be elevated in HCM [23] and both native T1 and ECV are elevated in genotype positive patients without overt hypertrophy [24, 25].

It is presently unknown which tissue characteristics are associated with contractile dysfunction in HCM. We therefore planned to assess native T1, ECV and LGE segmentally and quantify their association with strain measured in the same segment. This would provide insight into the pathological processes that lead to impaired contractility in HCM.

\section{Methods \\ Enrolment criteria}

Fifty consecutive patients with HCM were prospectively recruited from the local Inherited Cardiovascular Conditions Service between August 2014 and August 2015 and healthy controls $(N=30)$ were also recruited. The diagnosis of HCM was made independently by clinicians in keeping with current guidelines and based upon imaging including CMR, ECG, exercise testing, family history and genetic testing if possible $[1,8]$. Exclusion criteria were previous surgical myomectomy, previous septal ablation, atrial fibrillation, previous myocardial infarction, uncontrolled hypertension, permanent pacemaker, defibrillator or other contraindication to CMR. Healthy controls had no existing medical conditions and were not taking any regular medication were also recruited to establish the normal range of ECV using the identical CMR protocol.

\section{CMR protocol}

All subjects underwent an identical CMR protocol performed on a 3.0 Tesla Philips Achieva TX system (Philips, Best, The Netherlands) equipped with a 32 channel cardiac phased array receiver coil. A full blood count, including haematocrit was measured at the time of intravenous cannulation. The cardiac long and short axes were determined using standard scout views. Basal, mid and apical pre-contrast (native) short axis T1 maps were generated using a validated Modified Look Locker Inversion (MOLLI) protocol [26] (ECG triggered 5b (3 s) 3b MOLLI scheme with voxel size of $1.98 \times 1.98 \mathrm{~mm}^{2}$, slice thickness $10 \mathrm{~mm}$ ) and were planned using the 3 of 5 method [27]. Tissue tagging using a spatial modulation of magnetization (SPAMM) pulse sequence (spatial resolution $1.51 \times 1.57 \times 10 \mathrm{~mm}^{3}$, tag separation $7 \mathrm{~mm}, \geq 18$ phases, typical TR/TE $5.8 / 3.5 \mathrm{~ms}$, flip angle $10^{\circ}$, typical temporal resolution $55 \mathrm{~ms}$ ) was acquired in the same three short axis slices in 34/50 patients. Left ventricular volumes were obtained from cine imaging covering the entire LV in the short axis: balanced steady state free precession (SSFP), voxel size $1.2 \times 1.2 \mathrm{~mm}^{2}$, slice thickness $10 \mathrm{~mm}$ with no gap, 50 cardiac phases. Left atrial (LA) volumes were obtained from cine imaging covering the entire heart in the transverse axis: balanced SSFP, 
voxel size $1.2 \times 1.2 \mathrm{~mm}^{2}$, slice thickness $6 \mathrm{~mm}$ with no gap, 50 cardiac phases. $0.15 \mathrm{mmol} / \mathrm{Kg}$ Gadovist (Bayer Schering) was delivered by power injector (Medrad Inc, Warrendale, Pennsylvania, USA) as a single bolus via a cannula placed in the ante-cubital fossa followed by $20 \mathrm{ml}$ saline flush. Typical parameters for LGE were TR/TE 3.5/ $2.0 \mathrm{~ms}$, flip angle $25^{\circ}$, acquired spatial resolution $1.54 \times 1.76 \times 10 \mathrm{~mm}^{3}$ and performed in $10-12$ short axis slices with $\geq 3$ long axis orientations and phase-swapped acquisitions if indicated. Post contrast T1 mapping, using the same $5 \mathrm{~b}(3 \mathrm{~s}) 3 \mathrm{~b}$ MOLLI scheme as for native T1 mapping, was carried out in the same three slices exactly 15 min following last contrast injection (as above).

\section{CMR interpretation}

Analysis was carried out using CVI42 (Circle Cardiovascular Imaging Inc. Calgary, Canada) and inTag (v1.0, CREATIS lab, Lyon, France) by two physicians blinded to clinical data. LV mass, end diastolic volumes (EDV), end systolic volume (ESV) and LV ejection fraction (EF) were measured from short axis cine images excluding papillary muscles and trabeculations. Native and post contrast T1 relaxation time of myocardium and blood pool were measured from the three scanner generated T1 maps by contouring a region of interest in each segment of the 16 segment American Heart Association (AHA) model [28]. ECV was calculated from native and post contrast T1 times of myocardium and blood pool and haematocrit as previously reported [19]. Segment thickness was measured for each AHA segment from the identical end-diastolic SSFP cine images corresponding to the T1 maps. Feature tracking analysis was carried out on the same images by drawing endocardial and epicardial contours and circumferential (Ecc-FT) and radial (Err-FT) strain calculated for each AHA segment. The reproducibility of Ecc-FT and Err-FT tested in $30 \mathrm{seg}$ ments from 6 patients was good (intraobserver Ecc-FT 11.0\%, Err-FT 7.7\% and interobserver Ecc-FT 13.4\%, Err-FT 10.1\%).

For tagging analysis endocardial and epicardial contours were drawn on the short axis SPAMM sequences. Peak circumferential strain was measured for each segment of the AHA model using inTag (v1.0, CREATIS lab, Lyon, France). Strain was measured in the midmyocardial layer (by disregarding epicardial and endocardial layers) which has previously been reported to be the most reproducible [10].

Segments were defined as hypertrophied if the maximal thickness was $\geq 15 \mathrm{~mm}$ in keeping with current guidelines [1, 8]. Replacement fibrosis was defined on a binary scale as the presence of LGE reported by 2 physicians experienced in CMR interpretation for each segment. All analyses were carried out blinded to the results of strain data.

\section{Statistical analysis}

Statistical analysis was performed using Stata 14 (StataCorp, 2015, College Station, TX). Continuous variables are expressed as means \pm standard deviation (SD). Categorical variables are expressed as N (\%). Shapiro-Wilk test was used to test normality then unpaired $T$ test or Mann Whitney $U$ test used as appropriate. A mixed effects model with random intercept for each person was used to assess the association between Ecc-FT, Err-FT and Ecc-SPAMM and segment thickness, native T1, ECV and LGE. For each patient 16 segments with each parameter were included in the model to include segments with varying degrees of phenotypic expression. This model was used to account for the fact that segments from the same patient may not behave independently. The association between each strain parameter and segment thickness, native T1, ECV and LGE was tested using a two multivariable mixed effects model with random intercept adjusting for all three independent variables. ECV is not statistically independent from native $\mathrm{T} 1$ and therefore separate multivariable models were tested for each. Results are reported as mean \pm standard deviation. $P<0.05$ was considered statistically significant.

\section{Results}

Fifty patients with HCM were recruited. Patient characteristics are shown in Table 1. The distribution of hypertrophy was asymmetrical septal $36(72 \%)$, concentric 5 $(10 \%)$, mid cavity $4(8 \%)$, apical $3(6 \%)$ and isolated lateral 2 (4\%). An example of cine imaging, native T1, LGE and ECV mapping from an identical slice of one patient is shown in Fig. 1. Segment thickness, LGE and tissue tagging could be analysed in all available segments. Native T1 could be analysed in 781/800 segments (median $1227 \mathrm{~ms}$, interquartile range 1176-1271 ms) and ECV in 756/800 segments (median 27.4\%, interquartile range $25.0-31.6 \%$ ). Ecc-FT could not be analysed in $17 / 800$ and Err-FT in 12/800 because of either artefact or poor tracking of myocardial features.

Controls were $36.2 \pm 11.6$ years old and 22 (73\%) were male. From these controls the normal range for native T1 was $1190 \pm 24.7 \mathrm{~ms}$ and ECV $24.3 \pm 2.6 \%$. Therefore segments with native $\mathrm{T} 1>1239.4 \mathrm{~ms}$ and $\mathrm{ECV}>29.5 \%$ $(+2 \mathrm{SD})$ were defined as abnormally elevated.

The presence of hypertrophy, LGE, raised native T1 and raised ECV were all associated with impairment of all Ecc-FT, Err-FT and Ecc-SPAMM $(P<0.001$ for all), Table 2.

\section{Mixed effects model with random intercept}

On univariable analysis segment thickness, LGE, native T1 and ECV all had a significant association with Ecc-FT, Err-FT and Ecc-SPAMM, Table 3. In multivariable model 1 (segment thickness, LGE, ECV) only segment 
Table 1 Patient characteristics and findings

\begin{tabular}{|c|c|c|c|}
\hline $\mathrm{N}$ & 50 & FH of HCM, n (\%) & $19(38)$ \\
\hline Male gender, n (\%) & $37(74)$ & CMR findings & \\
\hline Age, years & $46.9 \pm 11.7$ & LV EDV, $\mathrm{ml} / \mathrm{m}^{2}$ & $78.7 \pm 13.1$ \\
\hline Height, cm & $171.2 \pm 9.0$ & LV EF, \% & $62.3 \pm 5.9$ \\
\hline Weight, kg & $81.7 \pm 14.6$ & LV Mass, g/m2 & $72.8 \pm 26.3$ \\
\hline Body mass index, $\mathrm{kg} / \mathrm{m}^{2}$ & $27.7 \pm 3.7$ & $\mathrm{LAV}, \mathrm{ml} / \mathrm{m}^{2}$ & $59.3 \pm 13.5$ \\
\hline Systolic blood pressure, $\mathrm{mmHg}$ & $125.5 \pm 17.6$ & LGE, n (\%) & $35(70)$ \\
\hline Diastolic blood pressure, $\mathrm{mmHg}$ & $74.5 \pm 12.5$ & Medications & \\
\hline Heart rate & $60.6 \pm 10.2$ & Beta blocker, n (\%) & $24(48)$ \\
\hline Echocardiography & & CC blocker, n (\%) & $8(16)$ \\
\hline Maximum wall thickness ${ }^{\mathrm{a}}(\mathrm{mm})$ & $19.3 \pm 4.9$ & Disopyramide, n (\%) & $4(8)$ \\
\hline LVOT obstruction, n (\%) & $11(22)$ & Diuretic, n (\%) & $2(4)$ \\
\hline Resting LVOT gradient, mmHg & $68.5 \pm 40.0$ & & \\
\hline
\end{tabular}

CC calcium channel, EDV end diastolic volume indexed to body surface area, EF ejection fraction, $F H$ family history, LAV left atrial volume indexed to body surface area, $L G E$ late gadolinium enhancement, $L V$ left ventricle, $L V O T$ left ventricular outflow tract

an 5 subjects with apical or localized lateral hypertrophy maximum wall thickness is taken from CMR
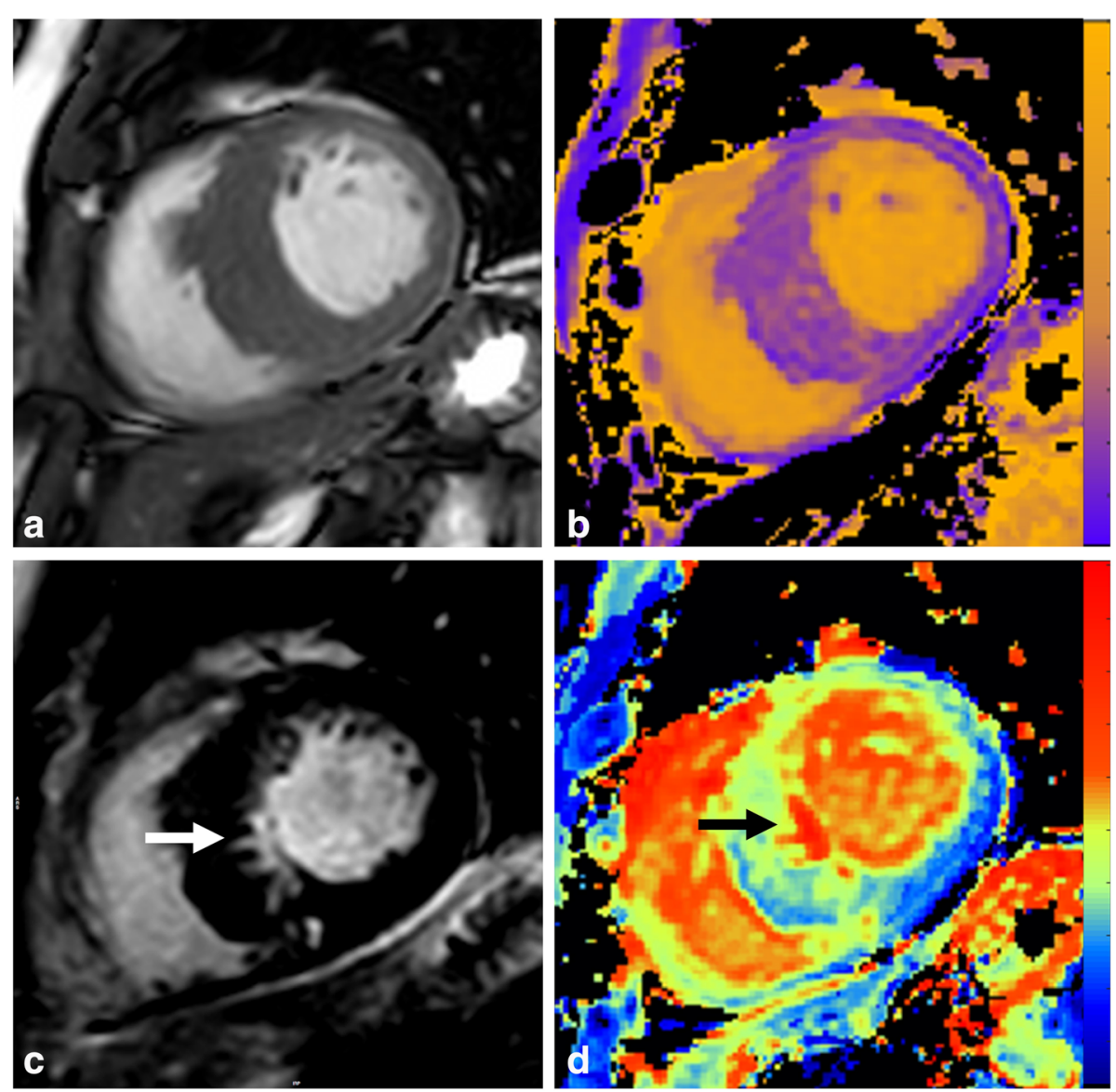

Fig. 1 CMR images from a patient with asymmetric septal HCM. a SSFP imaging showing gross septal hypertrophy (>15 mm). b Native T1 map with colour scale ranging from 0 (purple) to 2000 ms (yellow). c Late gadolinium enhancement imaging showing a discrete area of replacement fibrosis in inferoseptum (white arrow). d ECV map ranging from 0 (blue) to 100\% (red) confirming replacement fibrosis (black arrow) 


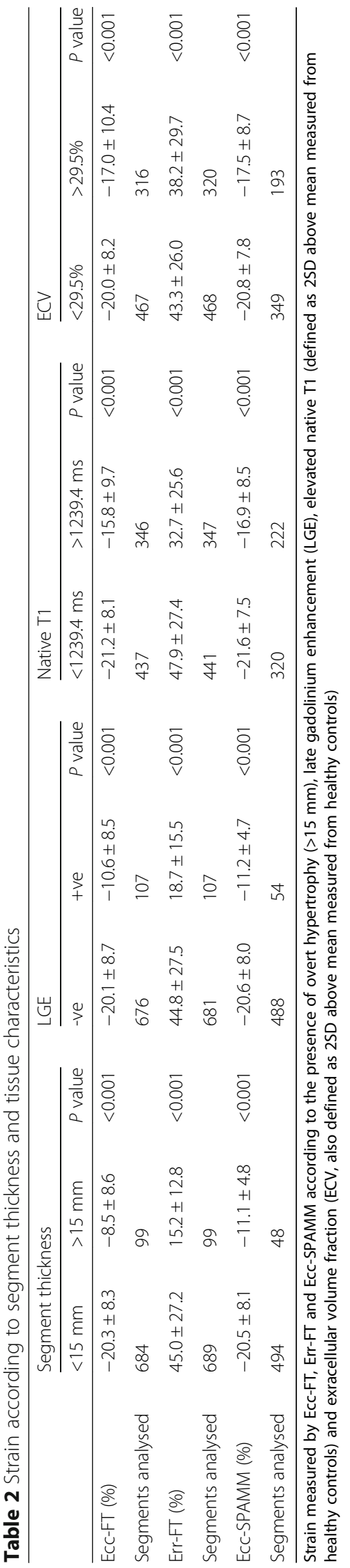




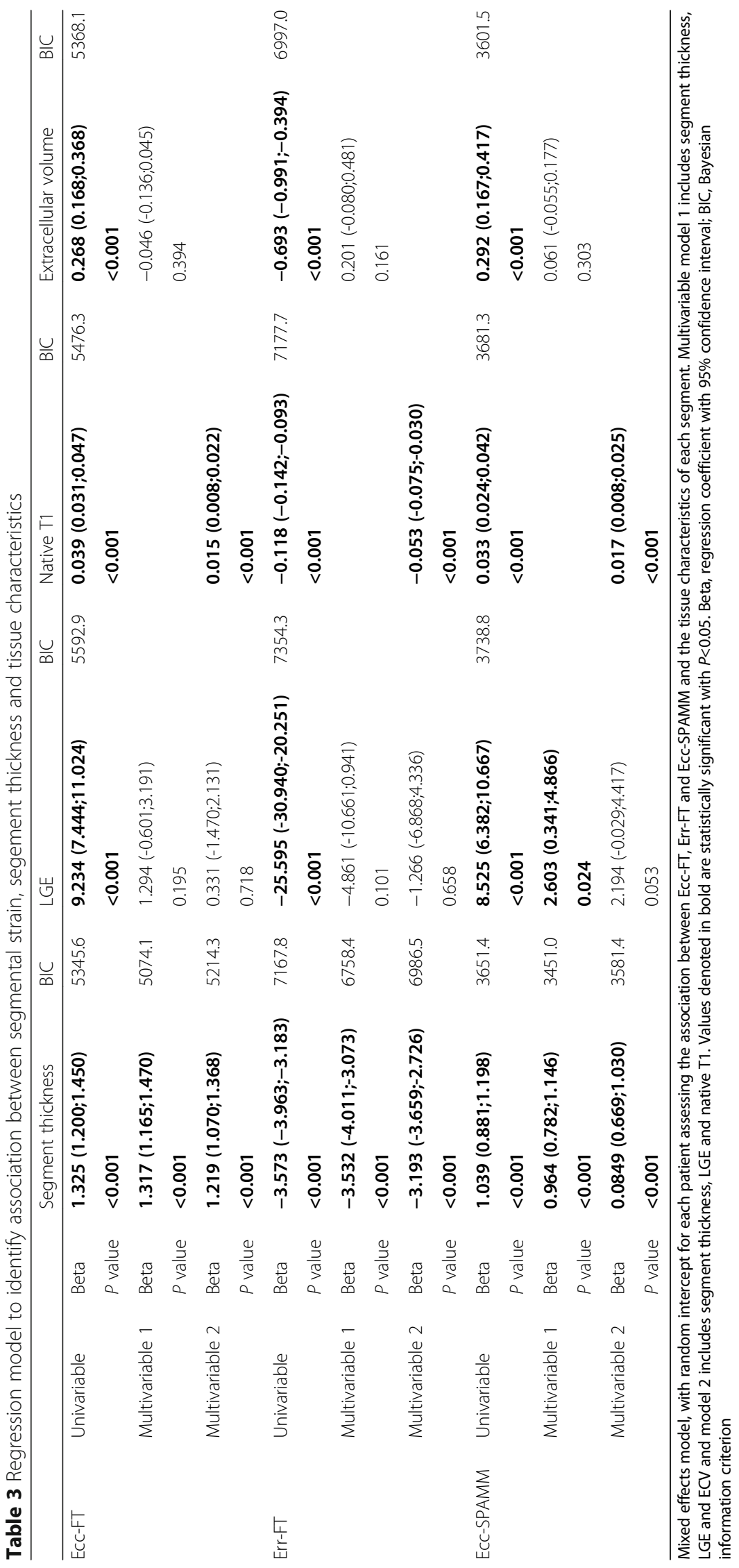


thickness had a significant association with Ecc-FT (Beta 1.317 (95\% confidence interval (CI) 1.165;1.470), $P<0.001$ ). In multivariable model 2 (segment thickness, LGE, native T1) both segment thickness (Beta 1.219 (95\% CI 1.070;1.368) $P<0.001$ ) and native T1 (Beta $0.015(95 \%$ CI $0.008 ; 0.022) P<0.001)$ had significant association with Ecc-FT.

In multivariable model 1 only segment thickness had a significant association with Err-FT (Beta -3.532 (95\% CI -4.011;-3.073), P < 0.001). In multivariable model 2 both segment thickness (Beta -3.193 (95\% CI (-3.659;-2.726) $P<0.001)$ and native T1 (Beta -0.053 (95\% CI -0.075;-0.030) $P<0.001)$ had significant association with Err-FT.

In multivariable model 1 both segment thickness (Beta 0.964 (95\% CI 0.782;1.146), $P<0.001$ ) and LGE (Beta 2.603 (95\% CI $0.341 ; 4.866), P=0.024$ ) had a significant association with Ecc-SPAMM. In multivariable model 2 both segment thickness (Beta 0.0849 (95\% CI 0.669;1.030) $P<0.001$ ) and native T1 (Beta 0.017 (95\% CI 0.008;0.025) $P<0.001)$ but not LGE had significant association with Ecc-SPAMM.

\section{Influence of genotype}

$10 / 50$ patients had presence of a genetic mutation known to cause HCM (MYBPC3 $N=7$, MYH7 $N=4$, TNNI3 $N=1)$. There was a trend to higher native T1 overtly hypertrophied segments $(>15 \mathrm{~mm}, N=99)$ in genotype positive vs genotype negative patients, $1295.9 \pm 65.1$ vs $1272.3 \pm 57.9 \mathrm{~ms}, P=0.055$. In the same segments there was no significant difference in ECV in genotype positive vs genotype negative patients, $37.1 \pm 10.1$ vs $34.8 \pm 10.2 \%$, $P=0.23)$.

\section{Discussion}

Hypertrophic cardiomyopathy is characterised by both cellular and extracellular pathological processes. With CMR it is possible to assess both gross macroscopic abnormalities, as reflected by segment thickness and replacement fibrosis on LGE, as well both the cellular and extracellular compartments at a microscopic level, by assessing ECV and native T1.

We have demonstrated that segmental contractility after correction for segment thickness has a significant association with myocardial native T1 but not LGE or ECV. ECV measurement assesses predominantly the extracellular compartment, whereas native $\mathrm{T} 1$ reflects the intracellular water, iron and lipid content [22]. These findings suggest that in HCM changes in the cellular structure and function rather than extracellular expansion may mediate impairment of myocardial contractility.

The cellular mechanisms that lead from mutation of sarcomeric proteins to increased native $\mathrm{T} 1$ and impaired contractile function remain to be established [5]. Native T1 could help elucidate these mechanisms by identifying the extent of cellular changes in patients with different disease causing mutations. It also has the potential to be a marker for monitoring disease progression, response to clinical intervention and long-term prognosis.

Ecc-SPAMM is the best validated CMR method for the assessment of strain [29, 30]. However it is hampered by tag fading in diastole and the relatively coarse tag separation of $7 \mathrm{~mm}$ may limit use in thinned regions of myocardium. We have therefore also assessed strain by FT which - although less well validated than tissue tagging - overcomes the issues of tag fading and its spatial resolution of $1.2 \times 1.2 \mathrm{~mm}^{2}$ allows better assessment of thinned regions of myocardium than is possible with SPAMM. Both we and other groups have reported good reproducibility of strain measured by this method of FT. Reassuringly, both FT and SPAMM consistently demonstrated a relationship between native $\mathrm{T} 1$ in two orthogonal planes (circumferential and radial).

\section{T1 mapping}

Cellular changes that may be detected in HCM include altered calcium cycling, impaired biomechanical stress sensing and disturbed cardiac energy homeostasis [31] and extracellular changes include myocyte disarray and fibrosis [32, 33]. The assessment of both native T1 and ECV by CMR offer the unique opportunity to assess changes in both the cellular and extracellular compartments non-invasively. ECV measured by T1 mapping has shown to be elevated in HCM and has a strong correlation with fibrosis measured histologically [19, 34].

There is already clear evidence that in HCM both native T1 [23, 35] and ECV [19, 36] are elevated. This increase can even be detected in those with sarcomeric mutations but without overt hypertrophy [24]. We have observed that for all three measures of regional strain native $\mathrm{T} 1$ but not ECV was associated with contractile function suggesting that changes in cellular rather than extracellular tissue characteristics contribute predominantly to loss of contractile function in HCM. An alternative explanation is that native $\mathrm{T} 1$ is a stronger marker of fibrosis than ECV. However this seems less likely given that native T1 reflects intracellular as well as extracellular signal and studies have demonstrated that native T1 has a much weaker association with histological measures of extracellular fibrosis than $\operatorname{ECV}[37,38]$.

The major shortcoming of native $\mathrm{T} 1$ is that it varies significantly between field strengths, scanner vendor and technique used to measure it [39] and in clinical practice requires validation for the specific pulse sequence and field strength used [18]. We have previously reported excellent reproducibility in both phantom and human studies of the pulse sequence used in this study [40]. However native T1 has merits that would support its use in this clinical application. Unlike ECV its measurement 
does not require intravenous cannulation, administration of contrast, or a blood sample to measure haematocrit.

\section{Late gadolinium enhancement}

We have reported, from multivariable model 2, that native $\mathrm{T} 1$ but not LGE has a significant association with impairment of regional function. LGE, like ECV, is directly influenced by the extent of extracellular fibrosis with a strong linear correlation between the extent of LGE within a particular segment and the amount of collagen measured histologically [14].

We identified that LGE, even after correction for segment thickness, had a significant association with Ecc-SPAMM (multivariable model 1) in keeping with previous studies which did not include T1 mapping $[6,7]$. However, when native T1 was included (multivariable model 2) the association was no longer significant suggesting again that changes in cellular structure are more determinant in regional contractility.

In $\mathrm{HCM}$, there is a strong correlation between extent of LGE and ECV expansion [36] although the association between native T1 and LGE is much weaker [25]. Increased native $\mathrm{T} 1$ can be detected in regions without LGE $[35,41]$ and is therefore a good discriminator between healthy and diseased myocardium [23]. The fact that native T1 influences contractile function but LGE does not adds to the existing literature in demonstrating that it is able to measure cellular changes that we have previously been unable to quantify.

\section{Segment thickness}

We have also reported that segmental strain is strongly associated with segment thickness. These findings corroborate previous studies that have shown a relationship between degree of hypertrophy and presence of LGE and strain measured by speckle tracking echocardiography $[6,7]$ and feature tracking by CMR [42]. Dhillon et al. measured longitudinal strain by feature tracking echocardiography in subjects with HCM undergoing surgical myomectomy, also reporting histological findings and in vitro contractility of the surgically excised myocardium. They found that the degree of histological fibrosis correlated with both strain measured by echocardiography and in vitro [43]. However strain was measured in segments with severe hypertrophy causing LVOT obstruction and more than half of the specimens studied displayed small intramural coronary arteriole dysplasia which is known to correspond strongly with focal LGE detected by CMR [44]. Therefore the subjects studied were at the severe end of the hypertrophic spectrum and not directly comparable with subjects and segments in the present study with a wide range of phenotypic presentations.

\section{Limitations}

The patients studied all had an established HCM with a diagnosis made according to current guidelines $[1,8]$. Our findings cannot therefore be extrapolated to those without overt expression of HCM. The correlation of both native $\mathrm{T} 1$ and ECV with cellular and extracellular changes has not been tested histologically in the study however but has been done in previous studies [19, 34].

We have only measured strain from short axis measurements specifically because the strength of the segmental analysis that we have carried out is based on the fact that T1 maps, SSFP cines and LGE acquisitions can all be carried out in the identical short axis plane. Comparing strain from long axis cines to ECV from short axis maps would add an unacceptable degree of error.

\section{Conclusions}

Regional strain impairment measured by feature tracking and SPAMM is predominantly associated with the degree of hypertrophy and native $\mathrm{T} 1$, but not extracellular fibrosis (LGE or ECV). These findings suggest that impairment of contractility in HCM may be mediated by mechanisms other than extracellular expansion that may include cellular changes in structure and function. The cellular mechanisms leading to increased native $\mathrm{T} 1$ and its prognostic significance remain to be established.

\section{Abbreviations}

CMR: Cardiovascular magnetic resonance; ECV: Extracellular volume; EDV: End diastolic volume; EDVI: End diastolic volume indexed to BSA; EF: Ejection fraction; ESV: End systolic volume; FT: Feature tracking; HCM: Hypertrophic cardiomyopathy; LGE: Late gadolinium enhancement; LV: Left ventricle; LVMI: Left ventricle mass indexed to BSA; MOLLI: Modified Look-Locker inversion recovery; SPAMM: Spatial modulation of magnetization; SSFP: Steadystate free precession; $\mathrm{T} 1: \mathrm{T} 1$ tissue relaxation time

\section{Acknowledgements}

We acknowledge the work of radiographers involved in this project including Gavin Bainbridge, Stephen Mhiribii, Margaret Saysell and Caroline Richmond.

\section{Funding}

PS (FS/12/88/29901) \& SP (FS/1062/28409) are funded by British Heart Foundation fellowships. This study was supported by the National Institute for Health Research Leeds Clinical Research Facility. The views expressed are those of the author (s) and not necessarily those of the NHS, NIHR or the Department of Health.

\section{Availability of data and materials}

Not applicable

\section{Authors' contributions}

PPS: Conception and design, analysis, interpretation of data, drafting of manuscript. AKM: Conception and design, analysis, interpretation of data, drafting of manuscript. BE: Analysis, critical and intellectual revision of manuscript. GRLW: Analysis, critical and intellectual revision of manuscript. PG: Critical and intellectual revision of manuscript. DAB: Interpretation of data, critical and intellectual revision of manuscript. DPR: Critical and intellectual revision of manuscript. TAM: Critical and intellectual revision of manuscript. LED: Critical and intellectual revision of manuscript. JRF: Critical and intellectual revision of manuscript. GJF: Critical and intellectual revision of manuscript. SPP: Critical and intellectual revision of manuscript. JPP: Critical and intellectual revision of manuscript. SP: Conception and design, interpretation of data, drafting of manuscript. All authors read and approved the final manuscript. 


\section{Authors' information}

None.

\section{Competing interests}

The authors declare that they have no competing interests.

\section{Consent for publication}

Not applicable.

\section{Ethics approval and consent to participate}

Ethical approval was given by the National Research Ethics Service Committee Yorkshire \& the Humber (14/YH/0126). All participants gave informed written consent.

\section{Author details \\ ${ }^{1}$ Multidisciplinary Cardiovascular Research Centre (MCRC) \& Leeds Institute of Cardiovascular and Metabolic Medicine, University of Leeds, Clarendon Way, Leeds LS2 9JT, UK. Division of Epidemiology and Biostatistics, Leeds Institute of Cardiovascular and Metabolic Medicine, University of Leeds, Leeds, UK. ${ }^{3}$ Department of Medical Physics and Engineering, Leeds Teaching Hospitals NHS Trust, Leeds, UK. ${ }^{4}$ Inherited Cardiovascular Conditions Service, Leeds General Infirmary, Leeds LS1 3EX, UK.}

Received: 10 January 2017 Accepted: 27 January 2017 Published online: 20 February 2017

\section{References}

1. Gersh BJ, Maron BJ, Bonow RO, et al. 2011 ACCF/AHA guideline for the diagnosis and treatment of hypertrophic cardiomyopathy: executive summary: a report of the american college of cardiology foundation/ american heart association task force on practice guidelines. Circulation. 2011;124:2761-96

2. Klues HG, Schiffers A, Maron BJ. Phenotypic spectrum and patterns of left ventricular hypertrophy in hypertrophic cardiomyopathy: morphologic observations and significance as assessed by two-dimensional echocardiography in 600 patients. J Am Coll Cardiol. 1995;26:1699-708.

3. Maron MS, Maron BJ, Harrigan C, et al. Hypertrophic cardiomyopathy phenotype revisited after 50 years with cardiovascular magnetic resonance. J Am Coll Cardiol. 2009;54:220-8.

4. Lopes LR, Elliott PM. A straightforward guide to the sarcomeric basis of cardiomyopathies. Heart. 2014;100:1916-23.

5. Watkins $H$, Ashrafian H, McKenna WJ. The genetics of hypertrophic cardiomyopathy: Teare redux. Heart. 2008:94(10):1264-8.

6. Urbano-Moral JA, Rowin EJ, Maron MS, et al. Investigation of global and regional myocardial mechanics with 3-dimensional speckle tracking echocardiography and relations to hypertrophy and fibrosis in hypertrophic cardiomyopathy. Circ Cardiovasc Imaging. 2014;7:11-9.

7. Popovic ZB, Kwon DH, Mishra M, et al. Association between regional ventricular function and myocardial fibrosis in hypertrophic cardiomyopathy assessed by speckle tracking echocardiography and delayed hyperenhancement magnetic resonance imaging. J Am Soc Echocardiogr. 2008;21:1299-305.

8. Elliott PM, Anastasakis A, Borger MA, et al. 2014 ESC guidelines on diagnosis and management of hypertrophic cardiomyopathy: the task force for the diagnosis and management of hypertrophic cardiomyopathy of the european society of cardiology (ESC). Eur Heart J. 2014;35:2733-79.

9. Donekal S, Ambale-Venkatesh B, Berkowitz S, et al. Inter-study reproducibility of cardiovascular magnetic resonance tagging. J Cardiovasc Magn Reson. 2013;15:37.

10. Swoboda PP, Larghat A, Zaman A, et al. Reproducibility of myocardial strain and left ventricular twist measured using complementary spatial modulation of magnetization. J Magn Reson Imaging. 2013;39(4):887-94.

11. Moody WE, Taylor RJ, Edwards NC, et al. Comparison of magnetic resonance feature tracking for systolic and diastolic strain and strain rate calculation with spatial modulation of magnetization imaging analysis. J Magn Reson Imaging. 2015;41:1000-12.

12. Schuster A, Stahnke VC, Unterberg-Buchwald C, et al. Cardiovascular magnetic resonance feature-tracking assessment of myocardial mechanics: intervendor agreement and considerations regarding reproducibility. Clin Radiol. 2015;70:989-98.
13. Pedrizzetti G, Claus P, Kilner PJ, et al. Principles of cardiovascular magnetic resonance feature tracking and echocardiographic speckle tracking for informed clinical use. J Cardiovasc Magn Reson. 2016;18:51.

14. Moon JC, Reed E, Sheppard MN, et al. The Histologic basis of late gadolinium enhancement cardiovascular magnetic resonance in hypertrophic cardiomyopathy. J Am Coll Cardiol. 2004:43:2260-4.

15. Todiere G, Aquaro GD, Piaggi $P$, et al. Progression of myocardial fibrosis assessed with cardiac magnetic resonance in hypertrophic cardiomyopathy. J Am Coll Cardiol. 2012:60:922-9.

16. Chan RH, Maron BJ, Olivotto I, et al. Prognostic value of quantitative contrast-enhanced cardiovascular magnetic resonance for the evaluation of sudden death risk in patients with hypertrophic cardiomyopathy. Circulation. 2014:130:484-95.

17. Green JJ, Berger JS, Kramer CM, et al. Prognostic value of late gadolinium enhancement in clinical outcomes for hypertrophic cardiomyopathy. JACC Cardiovasc Imaging. 2012;5:370-7.

18. Moon JC, Messroghli DR, Kellman P, et al. Myocardial T1 mapping and extracellular volume quantification: a society for cardiovascular magnetic resonance (SCMR) and CMR working group of the european society of cardiology consensus statement. J Cardiovasc Magn Reson. 2013;15:92.

19. Flett AS, Hayward MP, Ashworth MT, et al. Equilibrium contrast cardiovascular magnetic resonance for the measurement of diffuse myocardial fibrosis: preliminary validation in humans. Circulation. 2010;122:138-44.

20. White SK, Sado DM, Fontana M, et al. T1 mapping for myocardial extracellular volume measurement by CMR: bolus only versus primed infusion technique. JACC Cardiovasc Imaging. 2013;6:955-62.

21. Puntmann VO, Peker $E$, Chandrashekhar $Y$, et al. T1 mapping in characterizing myocardial disease: a comprehensive review. Circ Res. 2016;119:277-99.

22. Schelbert EB, Messroghli DR. State of the Art: clinical applications of cardiac T1 mapping. Radiology. 2016;278:658-76.

23. Puntmann VO, Voigt T, Chen Z, et al. Native T1 mapping in differentiation of normal myocardium from diffuse disease in hypertrophic and dilated cardiomyopathy. JACC Cardiovasc Imaging. 2013;6:475-84.

24. Ho CY, Abbasi SA, Neilan TG, et al. T1 measurements identify extracellular volume expansion in hypertrophic cardiomyopathy sarcomere mutation carriers with and without left ventricular hypertrophy. Circ Cardiovasc Imaging. 2013;6:415-22.

25. Hinojar R, Varma N, Child N, et al. T1 mapping in discrimination of hypertrophic phenotypes: hypertensive heart disease and hypertrophic cardiomyopathy: findings from the international T1 multicenter cardiovascular magnetic resonance study. Circ Cardiovasc Imaging. 2015;8.

26. Kellman $\mathrm{P}$, Arai $\mathrm{AE}, \mathrm{Xue} \mathrm{H}$. T1 and extracellular volume mapping in the heart: estimation of error maps and the influence of noise on precision. J Cardiovasc Magn Reson. 2013;15:56.

27. Messroghli DR, Bainbridge GJ, Alfakih K, et al. Assessment of regional left ventricular function: accuracy and reproducibility of positioning standard short-axis sections in cardiac MR imaging. Radiology. 2005;235:229-36

28. Cerqueira MD, Weissman NJ, Dilsizian V, et al. Standardized myocardial segmentation and nomenclature for tomographic imaging of the heart. A statement for healthcare professionals from the cardiac imaging committee of the council on clinical cardiology of the american heart association. Circulation. 2002:105:539-42.

29. Axel L, Dougherty L. MR imaging of motion with spatial modulation of magnetization. Radiology. 1989:171:841-5.

30. el Ibrahim SH. Myocardial tagging by cardiovascular magnetic resonance: evolution of techniques-pulse sequences, analysis algorithms, and applications. J Cardiovasc Magn Reson. 2011;13:36.

31. Frey N, Luedde M, Katus HA. Mechanisms of disease: hypertrophic cardiomyopathy. Nat Rev Cardiol. 2011;9:91-100.

32. Varnava AM, Elliott PM, Baboonian C, et al. Hypertrophic cardiomyopathy: histopathological features of sudden death in cardiac troponin T disease. Circulation. 2001;104:1380-4

33. Ho CY, Lopez B, Coelho-Filho OR, et al. Myocardial fibrosis as an early manifestation of hypertrophic cardiomyopathy. N Engl J Med. 2010;363:552-63.

34. Iles LM, Ellims $\mathrm{AH}$, Llewellyn $\mathrm{H}$, et al. Histological validation of cardiac magnetic resonance analysis of regional and diffuse interstitial myocardial fibrosis. Eur Heart J Cardiovasc Imaging. 2015:16:14-22.

35. Dass S, Suttie JJ, Piechnik SK, et al. Myocardial tissue characterization using magnetic resonance noncontrast $\mathrm{t} 1$ mapping in hypertrophic and dilated cardiomyopathy. Circ Cardiovasc Imaging. 2012;5:726-33. 
36. Kellman P, Wilson JR, Xue $\mathrm{H}$, et al. Extracellular volume fraction mapping in the myocardium, part 2: initial clinical experience. J Cardiovasc Magn Reson. 2012;14:64.

37. Miller $\mathrm{CA}$, Naish $\mathrm{JH}$, Bishop P, et al. Comprehensive validation of cardiovascular magnetic resonance techniques for the assessment of myocardial extracellular volume. Circ Cardiovasc Imaging. 2013;6:373-83.

38. de Meester de Ravenstein C, Bouzin C, Lazam S, et al. Histological validation of measurement of diffuse interstitial myocardial fibrosis by myocardial extravascular volume fraction from modified look-locker imaging (MOLLI) T1 mapping at 3 T. J Cardiovasc Magn Reson. 2015;17:48.

39. Raman FS, Kawel-Boehm N, Gai N, et al. Modified look-locker inversion recovery $\mathrm{T1}$ mapping indices: assessment of accuracy and reproducibility between magnetic resonance scanners. J Cardiovasc Magn Reson. 2013;15:64.

40. McDiarmid AK, Broadbent DA, Higgins DM, et al. The effect of changes to MOLLI scheme on T1 mapping and extra cellular volume calculation in healthy volunteers with 3 tesla cardiovascular magnetic resonance imaging. Quant Imaging Med Surg. 2015;5:503-10.

41. Ellims $A H$, lles $L M$, Ling $L H$, et al. A comprehensive evaluation of myocardial fibrosis in hypertrophic cardiomyopathy with cardiac magnetic resonance imaging: linking genotype with fibrotic phenotype. Eur Heart J Cardiovasc Imaging. 2014;15:1108-16.

42. Smith BM, Dorfman AL, Yu S, et al. Clinical significance of late gadolinium enhancement in patients $<20$ years of age with hypertrophic cardiomyopathy. Am J Cardiol. 2014;113:1234-9.

43. Dhillon A, Sweet W, Popovic ZB, et al. Association of noninvasively measured left ventricular mechanics with in vitro muscle contractile performance: a prospective study in hypertrophic cardiomyopathy patients. J Am Heart Assoc. 2014;3:e001269.

44. Kwon DH, Smedira NG, Rodriguez ER, et al. Cardiac magnetic resonance detection of myocardial scarring in hypertrophic cardiomyopathy: correlation with histopathology and prevalence of ventricular tachycardia. J Am Coll Cardiol. 2009;54:242-9.

\section{Submit your next manuscript to BioMed Central and we will help you at every step:}

- We accept pre-submission inquiries

- Our selector tool helps you to find the most relevant journal

- We provide round the clock customer support

- Convenient online submission

- Thorough peer review

- Inclusion in PubMed and all major indexing services

- Maximum visibility for your research

Submit your manuscript at www.biomedcentral.com/submit

) Biomed Central 